\title{
Charge spectrometry with a strongly coupled superconducting single-electron transistor
}

\author{
C. P. Heij, P. Hadley, and J. E. Mooij \\ Applied Physics and Delft Institute of Microelectronics and Submicron Technology, Delft University of Technology, Lorentzweg 1, 2628 \\ CJ Delft, The Netherlands \\ (Received 28 April 2001; revised manuscript received 25 June 2001; published 10 December 2001)
}

\begin{abstract}
We have used a superconducting single-electron transistor as a dc electrometer that is strongly coupled to the metal island of another transistor. With this setup, it is possible to directly measure the charge distribution on this island. The strong capacitive coupling was achieved by a multilayer fabrication technique that allowed us to make the coupling capacitance bigger than the junction capacitances. Simulations of this system were done using the orthodox theory of single-electron tunneling and showed excellent agreement with the measurements.
\end{abstract}

DOI: 10.1103/PhysRevB.64.245116

PACS number(s): 73.23.Hk, 85.35.Gv, 85.25.Na

A single-electron transistor can make extremely sensitive charge measurements with a resolution of about $10^{-5}$ $e / \sqrt{\mathrm{Hz}}{ }^{1-3}$ In principle, this sensitivity is sufficient to measure the charging and discharging of a small conductor as current flows through that conductor. A single-electron transistor would be able to register the tunneling of individual electrons tunnel onto the conductor from one lead and off the conductor to another lead for currents up to a few picoamperes. In practice, the high output impedance of a singleelectron transistor makes this measurement very difficult. If the charge on the conductor as a function of time could be measured, then it would be possible to determine the occupation probabilities of the various charge states. In charge state $\langle 0\rangle$ the conductor is neutrally charged, in charge state $\langle 1\rangle$ the conductor has an excess charge of one electron, and so forth. The charge state occupation probabilities play a central role in the orthodox theory of single-electron tunneling ${ }^{4,5}$ but are typically not directly accessible experimentally. Here we report on an experiment where a superconducting single-electron transistor (SSET) was used to directly measure the charge state occupation probabilities on the island of another nearby SSET.

An essential feature of this experiment was that the measurement SSET (the electrometer) was strongly coupled to the island where the current was flowing through. Strong coupling means that the coupling capacitance was comparable to the total capacitance of the nearby island. When one electron was added to this island, this induced a shift in the background charge of the electrometer island of about $e / 4$. The electrometer was biased at a small voltage and its tuning gate was used to scan the charge on the neighboring island. These gate traces directly reflect the charge distribution on this island. The measurements are consistent with orthodox theory and they show that a strongly coupled SSET can be used to directly measure the charge distribution.

\section{EXPERIMENT}

A schematic of the device is shown in Fig. 1(a). The device was fabricated in three layers. The junctions were fabricated using standard shadow evaporation of aluminum. SSET1 has a planar gate capacitor $C_{g 1}$, while the gate capacitor $C_{g 2}$ is defined as a parallel plate capacitor. Details of the fabrication of a similar device were described elsewhere. ${ }^{6}$ Figure 1(b) shows a scanning electron microscopy (SEM) picture of the device. The two square islands of the SSET's are coupled via an underlying dumbbell-shaped conductor. The coupling conductor itself is an island with a small capacitance, but because the number of electrons on the conductor does not change, it does not add an extra degree of freedom to the system. Consequently we can describe the system by only taking into consideration the excess electrons $n_{1}$ and $n_{2}$ on the two islands of the SSET's, coupled by a single effective capacitance. The total effective capacitance between the two islands is called $C_{m}$. Both SSET's were biased asymmetrically, connected to a voltage source at one side and grounded at the other side. The device was measured in a dilution refrigerator with a base temperature of 10 $\mathrm{mK}$. The leads were equipped with $\pi$ filters at room temperature and standard copper-powder filters ${ }^{7}$ at base temperature. The effective electron temperature, $25 \mathrm{mK}$, was mea-
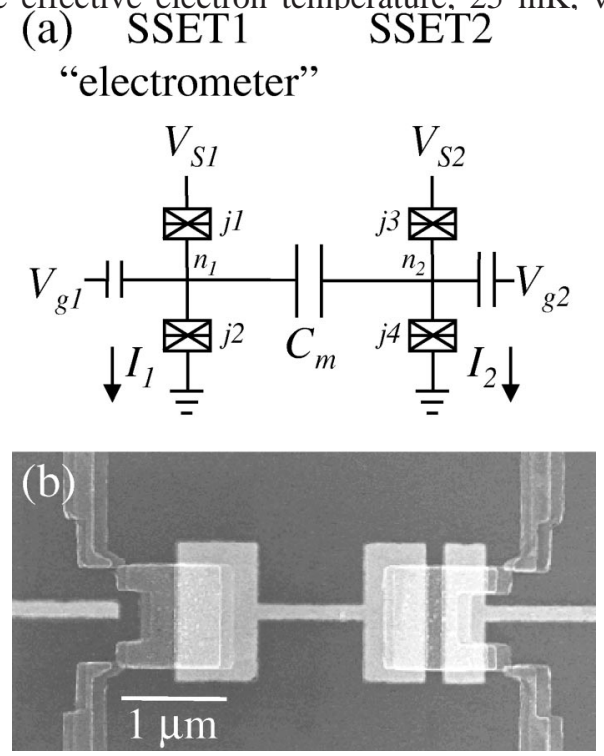

FIG. 1. (a) Schematic of the circuit. The two junctions on the left form the electrometer (SSET1), whose island is coupled capacitively to the island of a nearby SSET (SSET2). (b) Scanning electron microscope picture of the completed device. The light gray layer is fabricated in gold, and the aluminum layer shows up as dark grey. The bottom junctions are larger than the top junctions due to the proximity of a large electrode written $3 \mu \mathrm{m}$ underneath the bottom junctions. 


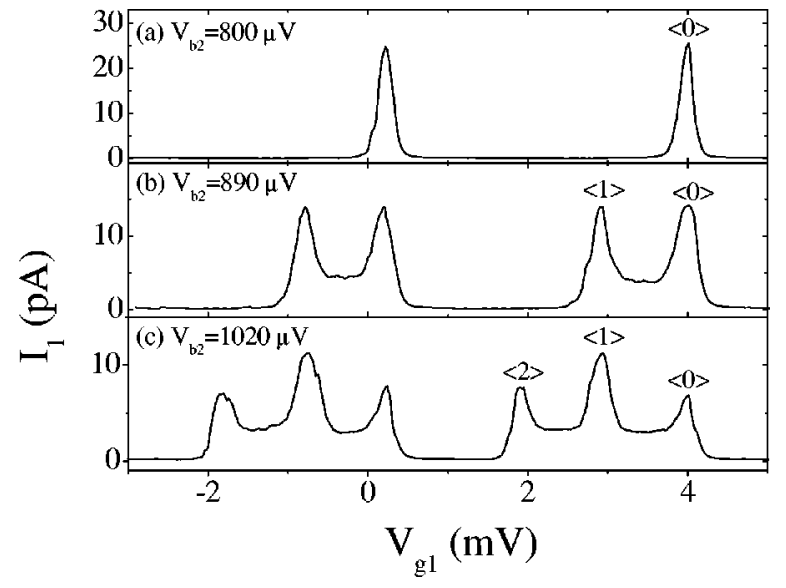

FIG. 2. Experimental Coulomb traces of the electrometer for different values of $V_{b 2}$ while $V_{b 1}=5 \mu \mathrm{V}, V_{g 2}=10 \mu \mathrm{V}$, and $T$ $=25 \mathrm{mK}$. The extra peaks Coulomb peaks in (b) and (c) correspond to the presence of extra electrons on island 2 .

sured in the normal state by fitting experimentally obtained Coulomb peaks. All further measurements were done in the superconducting state, the superconducting gap being $\Delta$ $=200 \mu e \mathrm{~V}$.

Throughout the measurements, the voltage bias of the electrometer was kept constant at $V_{b 1}=805 \mu \mathrm{V}$, just above $4 \Delta / e$. The current through the electrometer $I_{1}$ was measured as a function of the gate voltage $V_{g 1}$ and the bias voltage $V_{b 2}$ of the SSET2. The gate voltage $V_{g 2}$ was kept constant. Figure 2(a) shows typical Coulomb oscillations of the current through the electrometer. The gate of the electrometer was swept while SSET2 was biased at $800 \mu \mathrm{V}$, the current $I_{2}$ being negligibly small. Figure 2(b) shows the same Coulomb trace when $V_{b 2}=890 \mu \mathrm{V}$, above the quasiparticle threshold of SSET2. Surprisingly, the Coulomb peak is split into two peaks, while at even higher bias $\left(V_{b 2}=1090 \mu \mathrm{V}\right)$ it is split into three. As we will explain below, each extra peak can be attributed to the presence of an extra electron on the second island.

When $V_{b 2}=800 \mu \mathrm{V}$, the current through SSET2 is still negligibly small and the occupation probability of charge state $\langle 0\rangle$ on island 2 is nearly 1 . When $V_{b 2}$ is higher than the threshold voltage, a quasiparticle current $I_{2}$ will start to flow, and the charge $n_{2}$ on the island 2 will switch between $\langle 0\rangle$ and $\langle 1\rangle$. The presence of an extra electron on island 2 will induce a shift of a fraction of an electron in the background charge of island 1. By writing down the total charge on both islands as a function of the capacitances and the island potentials, one can show that this fraction is $C_{m} / C_{\Sigma 2}$, where $C_{\Sigma_{2}}$ is the sum of all capacitors connected directly to island 2 . Table I gives the capacitance and resistance values of all the circuit elements. With this table, we can calculate that the shift in background charge is $0.27 e$. This results in the extra Coulomb peak (labeled $\langle 1\rangle)$ shifted $-0.27\left(e / C_{g 1}\right)$ $=-1.04 \mathrm{mV}$ with respect to the peak labeled $\langle 0\rangle$ in Fig. 2(b). When $V_{b 2}$ is increased even more, charge state $\langle 2\rangle$ is also populated on island 2 and three peaks appear [Fig. 2(c)]. Figure 3 shows the Coulomb traces of the electrometer current $I_{1}$ in gray scale versus the bias $V_{b 2}$. One can clearly see
TABLE I. Capacitance and resistance values for the circuit parameters as calculated from the stability diagrams and currentvoltage characteristics of both SSETs.

\begin{tabular}{lccccccccc}
\hline \hline & $\mathrm{j} 1$ & $\mathrm{j} 2$ & $\mathrm{j} 3$ & $\mathrm{j} 4$ & $C_{g 1}$ & $C_{g 2}$ & $C_{m}$ & $C_{\Sigma 1}$ & $C_{\Sigma 2}$ \\
\hline$C(\mathrm{aF})$ & 135 & 350 & 160 & 400 & 42 & 640 & 450 & 977 & 1650 \\
$R(\mathrm{M} \Omega)$ & 3.5 & 3.5 & 6.5 & 6.5 & $\infty$ & $\infty$ & $\infty$ & - & - \\
\hline \hline
\end{tabular}

that consecutive charge states become populated with increasing $V_{b 2}$. The average bias voltage difference between successive charge states on island 2 is $2 E_{C 2} / e$, where $E_{C 2}$ is the charging energy of island 2 . The charge state $\langle 4\rangle$ becomes populated at $V_{b 2}=1180 \mu \mathrm{V}$ and induces $1.08 e$ on island 1 . The corresponding peak in the Coulomb trace of the electrometer overlaps with the next set of Coulomb peaks, limiting the number of observable charge states to four with these circuit parameters.

By measuring Coulomb oscillations, the electrometer can be used to directly resolve the average population of charge states on a nearby island, even though the charge on this island changes on a nanosecond time scale. At Coulomb peak $\langle j\rangle$, current can only flow through the electrometer when the island 2 is in charge state $\langle j\rangle$. The fraction of the time that island 2 spends in charge state $\langle j\rangle$ is equal to the relative peak height defined by

$$
p_{i}=\frac{I_{p, i}}{\sum_{j} I_{p, j}},
$$

where $I_{p, j}$ is the peak height of the Coulomb peak corresponding to the charge state $\langle j\rangle$ on island 2. Simulations confirm that the height of the individual peaks reflects the exact population of the corresponding charge state.

The relative peak heights of the Coulomb traces in Figs. 2(b) and 2(c) are calculated and shown in column I of Table II. They closely match the occupation of the various charge states as calculated in the simulations for identical bias con-

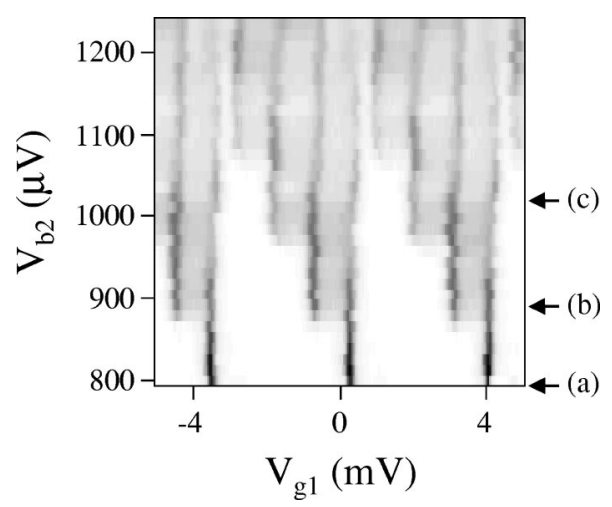

FIG. 3. Electrometer current versus $V_{g 1}$ and $V_{b 2}$. White indicates no current; black indicates a maximum current of $25 \mathrm{pA}$. The arrows indicate the values of $V_{b 2}$ where the traces of Figs. 2(a-c) have been extracted. At $V_{b 2}=1180 \mu \mathrm{V}$ the charge state $\langle 4\rangle$ becomes populated, but the corresponding peak overlaps with the neighboring set of Coulomb peaks. 
TABLE II. The population of first three charge states on island 2, as calculated from the peak heights in the experiments (Fig. 2) and the simulations (Fig. 4). The undisturbed population is determined by a calculation of the population matrix $P_{i j}$ of Eq. (2) when the electrometer is switched off $\left(V_{b 1}=800 \mu \mathrm{V}\right)$.

\begin{tabular}{lccccccccc}
\hline \hline & \multicolumn{4}{c}{ I. Experiments } & \multicolumn{4}{c}{ II. Simulations } & \multicolumn{3}{c}{ III. SSET1 “off” } \\
$V_{b 2}(\mu \mathrm{V})$ & $p_{0}$ & $p_{1}$ & $p_{2}$ & $p_{0}$ & $p_{1}$ & $p_{2}$ & $p_{0}$ & $p_{1}$ & $p_{2}$ \\
\hline 890 & 0.50 & 0.50 & 0 & 0.50 & 0.50 & 0 & 0.50 & 0.50 & 0 \\
1020 & 0.28 & 0.43 & 0.29 & 0.28 & 0.42 & 0.30 & 0.29 & 0.40 & 0.31 \\
\hline \hline
\end{tabular}

ditions, shown in column II. Also, for bias conditions other than $V_{b 2}=890 \mu \mathrm{V}$ and $1020 \mu \mathrm{V}$, the simulated relative peak heights closely match the experimental ones. This shows that a strongly coupled SSET can be used to quantitatively measure the charge distribution on a nearby object.

\section{SIMULATIONS}

The current through both SSET's was calculated using a master equation analysis. By solving the master equation we can calculate the occupation probabilities of the various charge states. The master equation for this two-island system is

$$
\begin{gathered}
\frac{\partial P_{i j}}{\partial t}=\sum_{k l \neq i j}\left(P_{k l} \Gamma_{k l \rightarrow i j}-P_{i j} \Gamma_{i j \rightarrow k l}\right), \\
\sum_{i j} P_{i j}=1,
\end{gathered}
$$

where $P_{i j}$ is the probability that the system has $i$ excess electrons on island 1 and $j$ excess electrons on island 2. $\Gamma$ denotes the transition rate between different charge states. In the stationary state, $P_{i j}$ does not change and the left hand side of Eq. (2) is zero. The first term on the right describes the population of charge state $i j$ from charge state $k l$, while the second term describes the depopulation of charge state $i j$ to charge state $k l$. We neglect co-tunneling processes and $\Gamma$ only is nonzero when either $i=k \pm 1$ or $j=l \pm 1$. Furthermore we only take into account a maximum of five charge states per island.

The superconducting tunnel rates $\Gamma$ were then determined with Fermi's golden rule using the superconducting density of states and the free energy difference $\Delta F$ of a tunneling event. ${ }^{8,9} \Delta F$ is the sum of the change in electrostatic energy plus the work done by the voltage sources. The total electrostatic energy of the system can be written as

$$
\begin{gathered}
E\left(n_{1}, n_{2}\right)=E_{C 1}\left(n_{01}+n_{1}\right)^{2}+E_{C 2}\left(n_{02}+n_{2}\right)^{2} \\
+E_{m}\left(n_{01}+n_{1}\right)\left(n_{02}+n_{2}\right), \\
E_{C 1}=\frac{e^{2} C_{\Sigma 2}}{2\left(C_{\Sigma 1} C_{\Sigma 2}-C_{m}^{2}\right)}=95 \mu \mathrm{eV},
\end{gathered}
$$

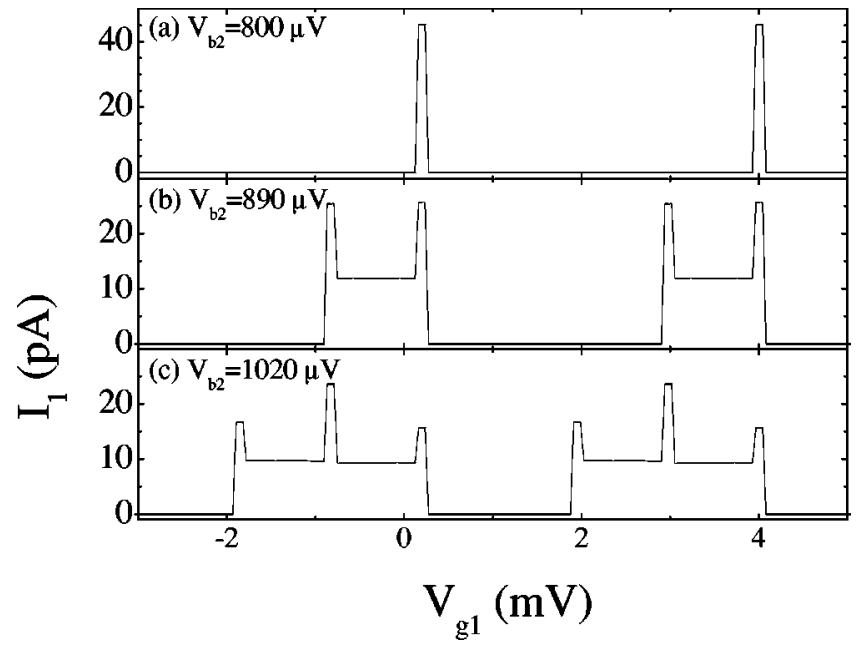

FIG. 4. Simulations of Coulomb traces of the electrometer for different values of $V_{b 2} . V_{b 1}=805 \mu \mathrm{V}, V_{g 2}=10 \mu \mathrm{V}$, and $\mathrm{T}=25 \mathrm{mK}$.

$$
\begin{gathered}
E_{C 2}=\frac{e^{2} C_{\Sigma 1}}{2\left(C_{\Sigma 1} C_{\Sigma 2}-C_{m}^{2}\right)}=53 \mu \mathrm{eV}, \\
E_{m}=\frac{e^{2} C_{m}}{C_{\Sigma 1} C_{\Sigma 2}-C_{m}^{2}}=51 \mu e \mathrm{~V},
\end{gathered}
$$

where $n_{01}$ and $n_{02}$ are the normalized charges induced on the islands by the voltage sources, $n_{1}$ and $n_{2}$ are the excess number of electrons on the islands, and $C_{\Sigma 1}$ and $C_{\Sigma 2}$ are the sum of all capacitors directly connected to the respective islands. The electrostatic energy has three contributions, the charging energies of the separate SSET's [Eqs. (5) and (6)], and the coupling energy $E_{m}$, which describes the electrostatic interaction between both SSET's. When Eqs. (2) and (3) are solved for $P_{i j}$, the total current $I_{1}$ can be calculated with $P_{i j}$ and the tunnel rates.

In Fig. 4 the current through the electrometer has been calculated for the same bias conditions as Fig. 2. One can clearly see the extra Coulomb peaks appear when the bias voltage $V_{b 2}$ is increased. The absolute peak height of the experiments is about $60 \%$ of the peak height in the simulations. This can be accounted for by the rounding of the superconducting gap. Instead of the discontinuous jump in quasiparticle current through a superconducting junction at $2 \Delta / e$, in real experiments, the current increases with a nonzero slope. In these experiments, the differential resistance in this regime is about $5 \%$ of the high bias junction resistance. For a bias voltage of $V_{b 1}=805 \mu \mathrm{V}$, only $5 \mu \mathrm{V}$ above $4 \Delta / e$, this has two consequences. First, the Coulomb peaks have a more triangular form as can be seen in Fig. 2, second, the Coulomb peak height is smaller than that in the simulations where the rounding has not been taken into account. Simulations where the rounding of the gap was taken into account with a simple model showed that the rounding of the gap does not change the relative height of the peaks; it merely decreases the overall current. 


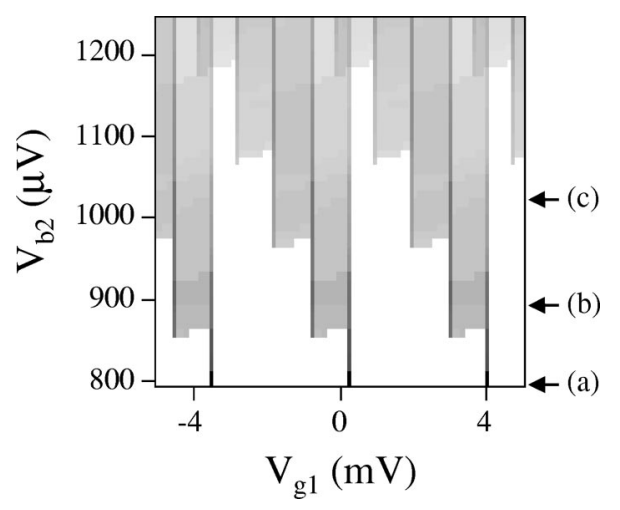

FIG. 5. Simulation of the electrometer current versus $V_{g 1}$ and $V_{b 2}$. White indicates no current; black indicates a current of $45 \mathrm{pA}$. The arrows indicate the values of $V_{b 2}$ where the traces of Fig. $4(\mathrm{a}-\mathrm{c})$ have been extracted.

For the simulated Coulomb traces of Fig. 4, the relative peak heights as specified by Eq. (1) are given in Table II. The experiments closely match the simulated values. The relative peak heights in simulations are slightly different though from the occupation of the charge states on island 2 when the electrometer is switched "off" ( $\left.V_{b 2}=800 \mu \mathrm{V}\right)$. Column III of Table II shows the undisturbed occupancies of the three charge states as determined from the population matrix $P_{i j}$. As can be seen from Table II, the bias of the electrometer has a small back action on the occupation of charge states on island 2. For the bias range of Fig. 5, it can be shown that the back action of this electrometer changes the occupancies of the various charge states by a maximum of $5 \%$.

Figure 5 is the simulated equivalent of Fig. 3. The extra peaks appear in Fig. 5 at exactly the same bias conditions as in Fig. 3, demonstrating the close agreement between experiments and simulations. Another feature that clearly shows up in the simulations as well as the measurements is the existence of a current plateau in between the neighboring Coulomb peaks. Under these bias conditions, the electrontunneling through both SSET's is correlated. This effect has been discussed for coupled one-dimensional (1D) arrays of tunnel junctions, but has never been demonstrated experimentally. ${ }^{10,11}$ The details of this effect will be discussed below.

\section{DISCUSSION}

The ability to determine the position and the height of the extra Coulomb peaks gives constraints on the bias conditions. In general, the width of the peaks has to be smaller than the separation between adjacent peaks. In the superconducting state the width of Coulomb peaks is almost independent of temperature for $k_{B} T<0.5 \Delta$ and depends linearly on the applied bias. This constraint can be rewritten as

$$
e V_{b 1}-4 \Delta<E_{m}
$$

This simply states that the energy associated with the voltage bias has to be smaller than the coupling energy. Because of the quasiparticle threshold at $4 \Delta / e$, this constrains the bias voltage to $800 \mu \mathrm{V}<V_{b 1}<851 \mu \mathrm{V}$ for this sample. The
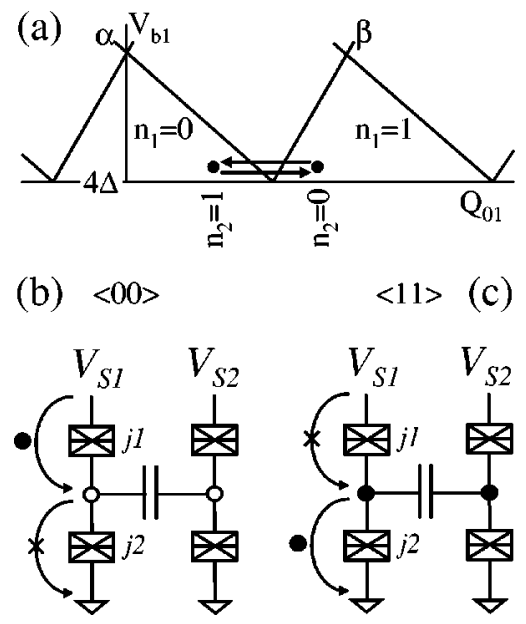

FIG. 6. (a) Schematic of the quasiparticle thresholds of SSET1 above $V_{b 1}=4 \Delta / e$, shown as thick lines. The positions of the dots denote the effective background charge induced by the absence or presence of an extra electron on island 2. (b) Visualization of the possible tunneling events on island 1 . When the system is in charge state $\langle 00\rangle$ it will decay to $\langle 10\rangle$ by an electron tunneling through junction $\mathrm{j} 1$. Electron tunneling through junction $\mathrm{j} 2$ is energetically unfavorable, just like electrons tunneling upward. (c) In a similar way charge state $\langle 11\rangle$ decays to $\langle 01\rangle$.

quasiparticle rate is almost independent of the bias in this bias window, and simulations indicate that the back action of the electrometer is also constant. If we take into account the rounding of the gap and the experimental current noise, $V_{b 2}=805 \mu \mathrm{V}$ is about the optimal bias voltage, combining an acceptable signal to noise ratio with a reasonably small width of the Coulomb peaks. With the current sample parameters, we are limited to the observation of a maximum of four charge states on the neighboring island. We estimate that it is feasible to observe at least seven different charge states, when the coupling capacitance is lowered to $190 \mathrm{aF}$, while keeping the other sample parameters constant.

Both Figs. 2 and 4 clearly show the existence of a current plateau in between the accompanying Coulomb peaks. In order to be able to measure the relative peak heights, this plateau current should not exceed the Coulomb peak current and therefore its mechanism should be understood. The mechanism can be most easily explained when the number of occupied electron states on island 2 is limited to two and under the assumption that the tunnel rates in SSET1 are much larger than those in SSET2. Figure 6(a) schematically displays the quasiparticle thresholds for SSET1. The position of the dots denotes the effective background charge when the charge state of island 2 is $\langle 0\rangle$ (right dot) and $\langle 1\rangle$ (left dot). The position of the dots relative to each other is fixed. The bias voltage $V_{b 1}$ and hence the dots lie just above $4 \Delta / e$. With the gate voltage $V_{g 1}$ the position of both dots can be shifted along the $Q_{01}$ axis. If the gate voltage positions one of the two dots above both quasiparticle thresholds $\alpha$ and $\beta$, this leads to current in the form of a Coulomb peak. If the dots are positioned as depicted in Fig. 6(a) there is an additional mechanism that will carry current. 
The charge states with the lowest energy are now $\langle 10\rangle$ and $\langle 01\rangle$. If a current is forced to flow through SSET2 by biasing it above its quasiparticle threshold, the following current cycle is most probable: If we start with charge state $\langle 00\rangle$ it is only favorable for electrons to tunnel onto islands 1 or 2 via the top junctions. Because we assume that the tunnel rates in SSET1 are much larger than those in SSET2, an electron will most probably tunnel through junction $\mathrm{j} 1$ first, as shown in Fig. 6(b). Now the system is in the charge state $\langle 10\rangle$, which is stable for electron tunneling in SSET1. After some time the bias voltage $V_{b 2}$ forces an electron on island 2 and the system is in state $\langle 11\rangle$. As can be seen in Fig. 6(c) this state decays to $\langle 01\rangle$ through junction $\mathrm{j} 2$, again assuming the tunnel rates are much higher in SSET1. This charge state is also stable for electron tunneling in SSET1. The cycle is completed when the electron is forced off island 2 and the system is back in charge state $\langle 00\rangle$. The cycle of one electron tunneling through SSET2 has transported another electron through SSET1, making $I_{1}=I_{2}$. This cycle is possible for all gate voltages where both the position of $n_{2}=\langle 1\rangle$ lies below quasiparticle threshold $\alpha$ and the position of $n_{2}=\langle 0\rangle$ lies below quasiparticle threshold $\beta$. This gives rise to a current plateau exactly between the Coulomb peaks attributed to the both charge states.

In this sample, the resistances of SSET1 and SSET2 and hence the tunnel rates differ by only a factor of 2 . This means that cycles can be missed, for example, if state $\langle 00\rangle$ decays to $\langle 01\rangle$, the system is forced to $\langle 00\rangle$. An electron is transported through SSET2, without giving rise to current in SSET1. The general equation for the relation between $I_{1}$ and $I_{2}$ can be deduced by analytically solving the master equation under the assumption that only the four charge states $\langle 00\rangle,\langle 10\rangle,\langle 01\rangle$, and $\langle 11\rangle$ need to be considered. If we assume that the tunnel rates through junction $\mathrm{j} 1$ and $\mathrm{j} 2$ are equal and called $\Gamma_{1}$, as well as those through $\mathrm{j} 3$ and $\mathrm{j} 4$ are equal and called $\Gamma_{2}$, this yields

$$
I_{1}=\frac{\Gamma_{1}}{\Gamma_{1}+2 \Gamma_{2}} I_{2} .
$$

By deriving the expressions for the Coulomb peak current, it can be shown that the peak currents are always sufficiently larger than the plateau current, making it possible to adequately determine the relative peak heights. When the number of occupied charge states on island 2 is larger than two, the mechanism leading to the current plateaus is similar, but different combinations of charge states might be stable and Eq. (9) will be modified. Again though, the plateau current is always smaller than the Coulomb peaks adjacent to the particular current plateau.

To check the validity of Eq. (9), we deduced the values of $\Gamma_{1}$ and $\Gamma_{2}$ from the peak heights of single Coulomb peaks; for example, see Fig. 2(a). The bias-dependent ratio $I_{1} / I_{2}$ that follows from Eq. (9) agreed with the experimentally measured current values.

We also studied the performance of the electrometer in the normal state. In the normal state, however, the Coulomb peaks are very sensitive to thermal fluctuations. The thermal broadening of the Coulomb peaks at $70 \mathrm{mK}$ was enough to merge the adjacent Coulomb peaks, making an accurate determination of the relative peak heights impossible. Additionally, due to a mechanism similar to the one leading to the plateau current in the superconducting state, the adjacent Coulomb peaks merged at $30 \mathrm{mK}$ in the normal state, making the normal state operation of this electrometer impractical.

\section{CONCLUSIONS}

We have used a SSET to measure the charge distribution on a neighboring island. Both the islands were strongly coupled by a multilayer technology. The presence of an extra electron on a neighboring island split the Coulomb peaks of the SSET. The relative height of these peaks directly translates to the occupation of the associated charge state. Between the neighboring Coulomb peaks the current is carried by correlated tunneling of electrons through both SSET's.

We thank K. K. Likharev for illuminating the basic mechanism leading to the current plateau.
${ }^{1}$ V. A. Krupenin, D. E. Presnov, A. B. Zorin, and J. Niemeyer, J. Low Temp. Phys. 118, 287 (2000).

${ }^{2}$ R. J. Schoelkopf, P. Wahlgren, A. A. Kozhevnikov, P. Delsing, and D. E. Prober, Science 280, 1238 (1998).

${ }^{3}$ E. H. Visscher, J. Lindeman, S. M. Verbrugh, P. Hadley, J. E. Mooij, and W. van der Vleuten, Appl. Phys. Lett. 68, 2014 (1996)

${ }^{4}$ D. V. Averin and K. K. Likharev, in Mesoscopic Phenomena in Solids, edited by B. L. Altshuler, P. A. Lee, and R. A. Webb (Elsevier, Amsterdam, 1991), p. 173.

${ }^{5}$ G. L. Ingold and Yu. V. Nazarov, in Single Charge Tunneling, edited by H. Grabert and M. H. Devoret (Plenum, New York, 1992), pp. 21-108.
${ }^{6}$ C. P. Heij, D. C. Dixon, P. Hadley, and J. E. Mooij, Appl. Phys. Lett. 74, 1042 (1999).

${ }^{7}$ J. M. Martinis, M. H. Devoret, and J. Clarke, Phys. Rev. B 35, 4682 (1987).

${ }^{8}$ See for example M. Tinkham, Introduction to Superconductivity, 2nd ed. (McGraw-Hill, New York, 1996), p. 77.

${ }^{9}$ Using Fermi's golden rule we numerically calculate the superconducting rates assuming the BCS density of states, which is proportional to $E /\left(E^{2}-\Delta^{2}\right)^{1 / 2}$.

${ }^{10}$ D. V. Averin, A. N. Korotkov, and Yu. V. Nazarov, Phys. Rev. Lett. 66, 2818 (1991).

${ }^{11}$ M. Matters, J. J. Versluys, and J. E. Mooij, Phys. Rev. Lett. 78, 2469 (1997). 